\title{
The advanced image slicers of OCTOCAM
}

Content, Robert, de Ugarte Postigo, Antonio, Thöne, Christina, Sheinis, Andrew

Robert Content, Antonio de Ugarte Postigo, Christina Thöne, Andrew Sheinis, "The advanced image slicers of OCTOCAM," Proc. SPIE 10706, Advances in Optical and Mechanical Technologies for Telescopes and Instrumentation III, 107066L (10 July 2018); doi: 10.1117/12.2314424

Event: SPIE Astronomical Telescopes + Instrumentation, 2018, Austin, Texas, United States 


\title{
The Advanced Image Slicers of OCTOCAM
}

\author{
Robert Content* $^{* \mathrm{a}}$, Antonio de Ugarte Postigo $^{\mathrm{b}}$, Christina Thöne ${ }^{\mathrm{b}}$, Andrew Sheinis ${ }^{\mathrm{c}}$ \\ ${ }^{a}$ Australian Astronomical Optics, 105 Delhi Road, North Ryde, NSW, Australia 2113; ${ }^{\mathrm{b}}$ Instituto de \\ Astrofísica de Andalucía - CSIC, Glorieta de la Astronomía s/n, 18008 Granada, Granada, Spain; \\ ${ }^{\mathrm{c} C}$ Canada-France-Hawaii Telescope, 65-1238 Mamalahoa Hwy, Kamuela, Hawaii 96743, USA
}

\begin{abstract}
OCTOCAM is the new large Gemini instrument in building. It is an imaging spectrograph with 8 cameras covering the range $370 \mathrm{~nm}$ to $2350 \mathrm{~nm}$ at a typical resolution of 3000-4000. It will have 2 IFUs, one for normal operation over all wavelengths, the other for AO in the NIR only and with a smaller field but a higher spectral resolution. Currently, no IFU exists that covers the entire range of VIS and NIR in a single observation. Such an IFU would have a number of applications: It can be used for resolved studies of HII regions over a broad wavelength range and emission line galaxies over a broad redshift range using the same set of emission lines. Another application is to observe transients with only arcseconds localization very early without waiting for a sub-arcsecond position, hence allowing to obtain very valuable early data. For bright transients such as SNe and GRBs we can study the immediate environment in detail, and even use the actual transient as AO tip-tilt star to study the environment at high spectral and very high angular resolutions. The IFUs will be Advanced Image Slicers, a proven concept now in use in many instruments around the world including Gemini NIFS, VLT MUSE and KMOS, and JWST NIRSpec. The normal operation slicer will have a field of 9.7" x 6.8" with 17 slices 0.4 " wide giving $0.18 "$ x 0.4 " spaxels. The slices are smaller than the standard slit size of 0.54 " ( 3 pixels) so will deliver higher spectral resolution. This IFU will deliver much higher performances than the GMOS IFU and NIFS with a larger field of view and spectral range but also considerably fewer pixels per $\operatorname{arsec}^{2}$ then reducing the readout noise. With its wavelength range starting at $370 \mathrm{~nm}$, diamond machining cannot be used. A glass slicer system will have to be used as in MUSE. The wavelength range will however be much larger covering the whole VIS and NIR range. Modern reflection coatings as UV enhanced silver can be used but a trade-off may be better by starting at a longer wavelength to get higher transmission. Special consideration is necessary for the fore-optics which cannot be diamond machined and for the overall design due to the limited space envelope. The AO slicer will have a field of 2.5 " x 3.6 " with 31 slices 0.08 " wide imaged on 2 pixels in the spectral direction to get proper sampling. The fore-optics will magnify the beam in both directions but with different magnifications to get spaxels of 0.08 " x 0.08 ". The smaller slice image width will give a spectral resolution of about 5000 including aberrations, about the same than NIFS but covering all 4 NIR bands at once. This slicer uses a slit $60 \%$ longer than OCTOCAM is designed for. It is possible because the magnification reduces the beam size so the aberrations and vignetting.
\end{abstract}

Keywords: Image slicer, integral field spectroscopy, VIS spectroscopy, NIR spectroscopy.

\section{INTRODUCTION}

Integral Field Spectroscopy (IFS) has become an inevitable part of almost any new spectrograph. Many even work only in that mode as MUSE $^{1}$ and NIFS ${ }^{2}$. Others have an Integral Field Unit (IFU) that can be moved into the input focal plane of the spectrograph as for the Gemini Multi-Object Spectrographs ${ }^{3}$ (GMOS) and the Inamori-Magellan Areal Camera and Spectrograph ${ }^{4}$ (IMACS), and the Gemini Near-Infrared Spectrograph ${ }^{5}$ (GNIRS; note that the GNIRS IFU was destroyed in the accident of the first GNIRS but a new one is presently being manufactured). Some finally, as $\mathrm{KMOS}^{6}$ (24 IFUs), SAMI (13 IFUs) and the future Hector ${ }^{8,9}$ (21 IFUs) combine Integral Field and Multi-Object Spectroscopy by having many IFUs that can be positioned on the sources in the field. GMOS and IMACS are for the extended visible region so their IFUs were chosen to be made of transparent optics since reflective optics were not particularly efficient in the ultra-violet and blue at the time it was designed. New reflection coatings developed more recently do have a reasonable reflectivity in the UV and blue. GNIRS being for the infrared, a reflective design was preferred. This design is based on the concept of Advanced Image Slicer ${ }^{10,11}$ (AIS) developed by the first author.

*robert.content@aao.gov.au; phone 61-2-9372-4846 


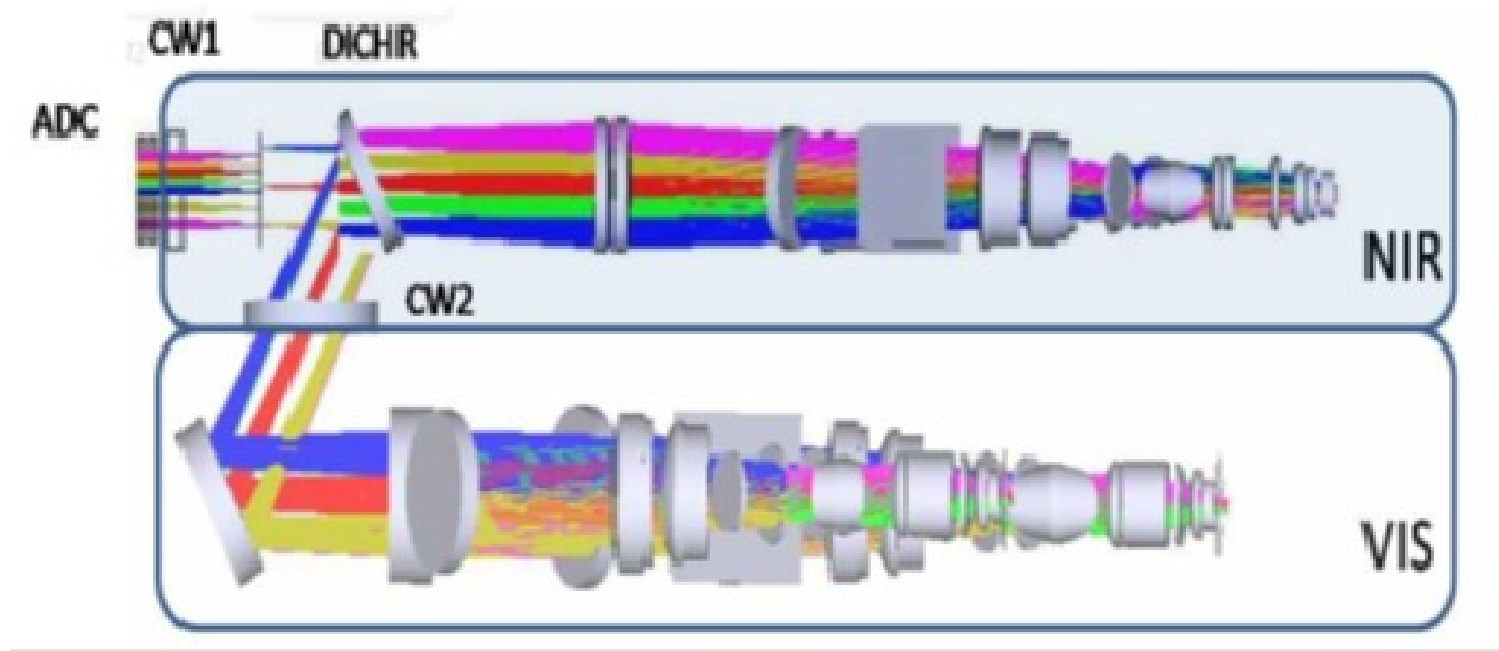

Figure 1. Side view of OCTOCAM layout.
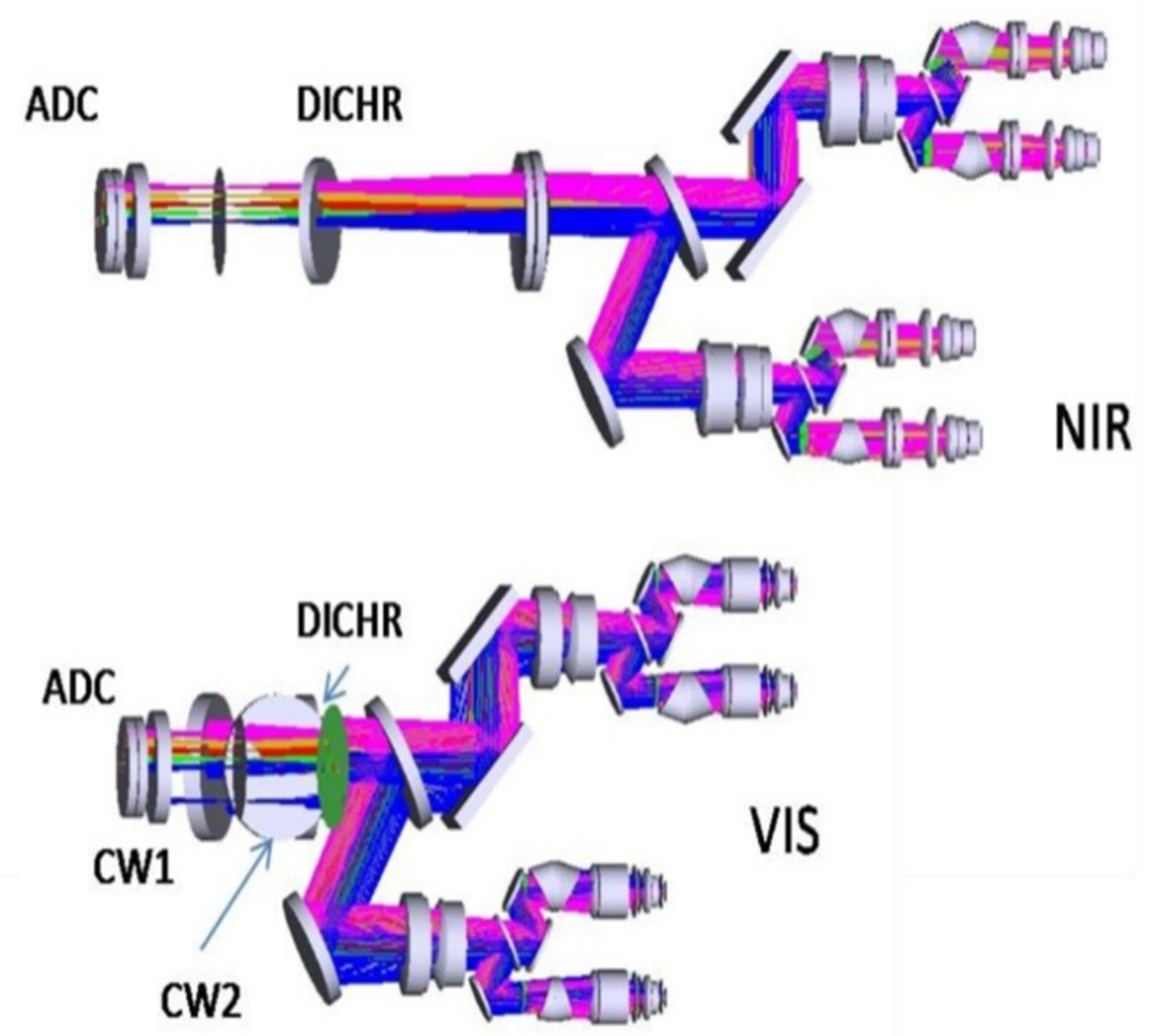

Figure 2. Top view of OCTOCAM layout.

OCTOCAM ${ }^{12}$ is the new large Gemini instrument being manufactured at the time of this paper (Figures $1 \& 2$ ). It is an imaging spectrograph with 8 cameras covering the range $370 \mathrm{~nm}$ to $2350 \mathrm{~nm}$ at a typical resolution of 3000-4000. In imaging mode, the field is nearly $3^{\prime}$ x $3^{\prime}$ while the slit length is nearly $3^{\prime}$ in spectroscopic mode. A dichroic split the light 
between VIS and NIR and directs each beam to a spectrograph. Further dichroics in the collimators and before the gratings split the light into a total of 8 bands. The NIR spectrograph and the focal plane area are in a cryostat while the VIS spectrograph is at ambient temperature. A slide in the telescope focal plane permits to position one of a series of instruments at the entrance of the spectrograph, namely a field mask, a slit box, a polarimeter box or an IFU box.

The IFU box will contain 2 IFUs, one with a large field and standard spatial resolution for seeing limited sources, and the other with a small field and high spatial resolution for Adaptive Optics (AO).

\section{OCTOCAM INTEGRAL FIELD UNITS}

Integral Field Units (IFUs) have been in use on telescopes in the past 20 years with great success. Gemini has the GMOS IFUs Nord and South, which are fiber-lenslets IFUs, and the NIFS spectrograph, which has an Advanced Image Slicer (AIS) in front of the spectrograph. An IFU on OCTOCAM will not only give the full spectrum from $0.37 \mu \mathrm{m}$ to $2.35 \mu \mathrm{m}$ in one observation but also give a larger field of view than these existing IFUs and a much lower detector noise due to the larger pixel solid angle in square arcseconds. It will also deliver a higher resolution than the smallest slit with minimal losses of light. All of this together will considerably increase the throughput and scientific usefulness of OCTOCAM.

OCTOCAM covers both the VIS and the NIR so this put new conditions on an IFU. In term of transmission, fiber systems are difficult to use in the NIR in particular because of the low transmission of standard fibers in the K band. Slicers on the other hand use mirrors so would need a good reflective coating for the whole of the VIS and NIR. Fortunately, coatings as Enhanced Silver now give an average reflectivity over the full VIS+NIR bandwidth of $>98 \%$. Combined with the higher performances of slicers compared to fiber systems with or without microlenses and microlens only systems which is extensively discussed in the literature, and the success of slicers on telescopes, a slicer system is the obvious choice.

The AIS has now been universally adopted and IFUs using this concept are on telescopes all over the world. Among them, KMOS and MUSE on the VLT both contain 24 AIS which are made of a total of more than 3300 mirrors the two together. The Keck telescope has an AIS named ESI-IFU ${ }^{13}$ and the JWST will contain 5 slicers $^{14,15}$ all using the basic design of the AIS or a simpler modified design but with lower performances. The very first AIS was however the GNIRS slicer which was accidentally destroyed with the first GNIRS. It worked perfectly well before the accident even giving a higher transmission than a slit of similar resolution over the wavelength range of 2 to $5 \mu \mathrm{m}$ due to losses from slit diffraction and the particularities of the slicer design. Most of these slicers are for the NIR but ESI and MUSE are for the VIS. The advantages of the AIS and the fact that it is a proven technology many times over - especially on Gemini which saw the first of them - for both the VIS and the NIR made it the chosen concept for the OCTOCAM IFU.

An important fact for an OCTOCAM slicer is that the ESI and MUSE slicers are in the VIS because the tolerances for the VIS are much tighter than for the NIR so the technology for the VIS should be the one used. The only additional challenge is that the IFU will be at much lower temperature than in ESI and MUSE because of its use in the NIR but this is not a critical mechanical problem for a system without moving parts. Also, a cryogenic test was made on ESI-IFU by putting it in liquid nitrogen which it successfully passed.

\subsection{Requirements}

The field of view should be larger than the existing fields of view of IFUs on Gemini instruments. NIFS has a field of 3" x $3 "$ and the GMOS IFUs have fields of 7" x $5 "$ in their largest configuration with 2 slits. The field of view of the OCTOCAM IFU is $9.7 " \mathrm{x} 6.8$ ".

Spatial sampling should be at least better than the seeing preferably not far from half the seeing to get a good sampling while maintaining a proper spectral sampling. The slice width is 0.4 " while the pixel size is 0.18 " so a spatial element is 0.18 " x 0.4 " which is not far from half the typical seeing in the largest direction. The slice width gives a spectral element 2.3 pixel wide which is larger than the minimum sampling of 2 pixel. This gives a resolution 1.35 times larger than the standard slit width of 0.6 " (3.3 pixel) including tolerances.

The transmission should be at least $80 \%$ on average and at least $50 \%$ at all wavelengths. There are 6 reflections in the IFU. Enhanced Silver coatings give an average reflectivity $>98 \%$ over the whole range so the average transmission should be $>89 \%$. The minimum reflectivity is at $370 \mathrm{~nm}$ but should be $>92 \%$ so the transmission should be $>60 \%$ at that wavelength. The GMOS IFUs have a transmission of about $63 \%$ while the NIFS IFU has a transmission around $95 \%$ 
(including fore-optics) so the OCTOCAM slicer will have a transmission higher than the average of the 2 instruments which is $79 \%$.

This however does not tell the whole story about the signal-to-noise ratio because both instruments are affected by the detector noise due to the small pixel sizes of these instruments. NIFS has pixels of $0.045^{\prime \prime} \times 0.05$ " while the GMOS IFU has pixels of 0.041". With pixels of 0.18" OCTOCAM will have 14 times less pixels than NIFS and 19 times less than the GMOS IFUs per square arcsecond on the sky. This will considerably reduce the detector noise.

The image quality, defined as the $50 \%$ EED, must be better than 0.15 " on average with a goal of $0.1 "$. The present design gives an average of $0.04 "$ to which the effect of the tolerances will be added. The tolerances will be adjusted accordingly.

\subsection{Basic design}

The AIS is made of fore-optics and 3 mirror arrays which are called the slicing mirror array, the pupil mirror array and the slit mirror array. The AIS slices the focal plane into many long and thin images that are reimaged side-by-side at the slit of the spectrograph (Figure 3). The width of the slice images then becomes the width of the slit.

\section{To spectrograph}

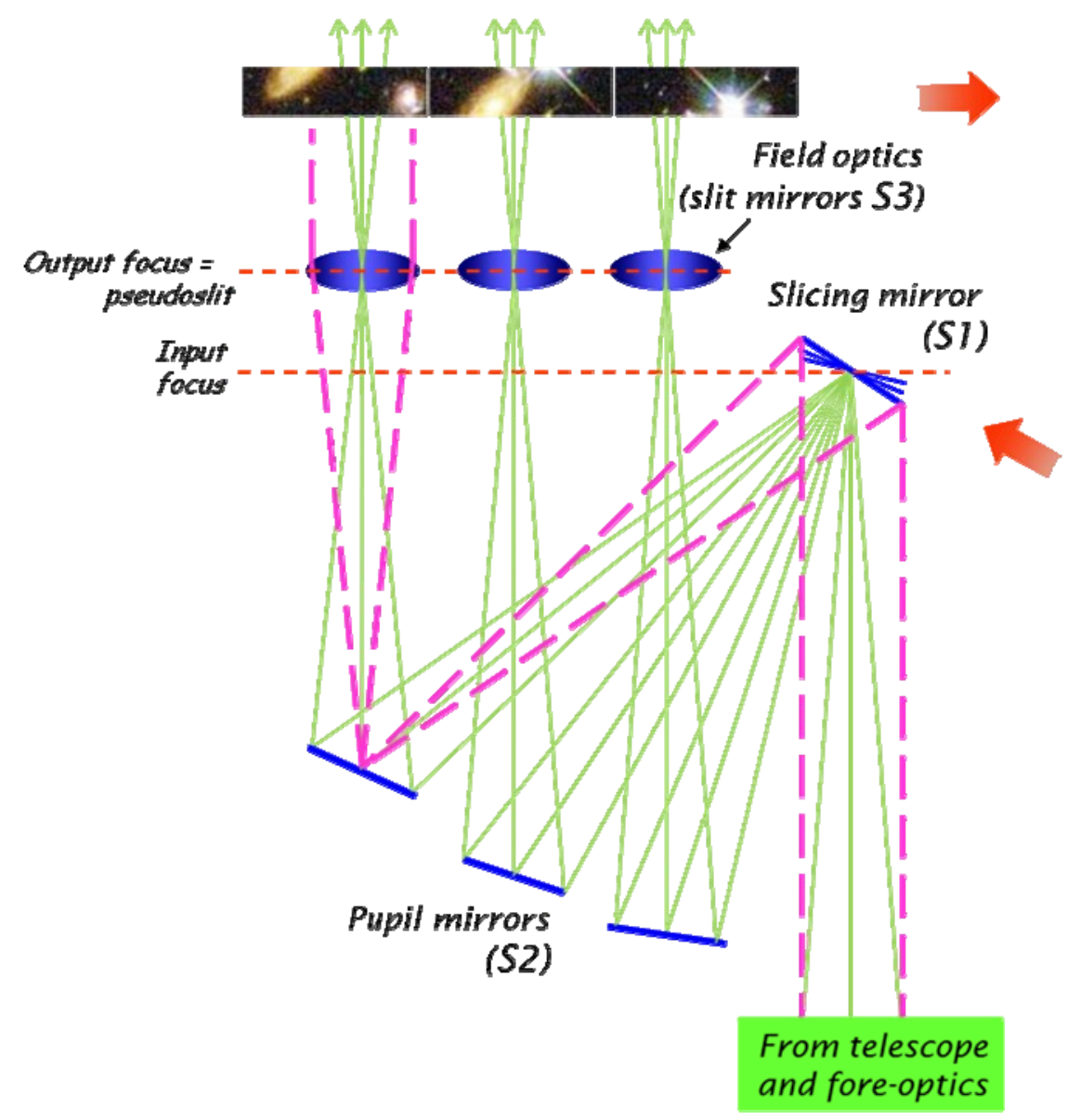

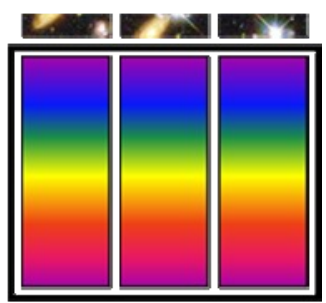

Spectrogram
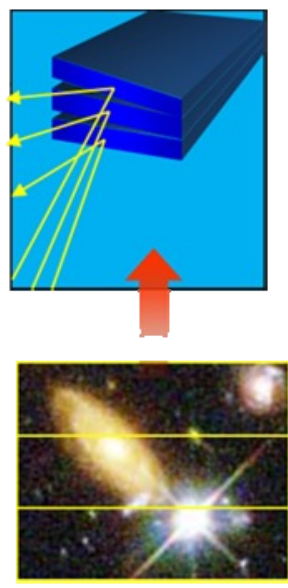

Field before slicing

Figure 3. Basic principle of the Advanced Image Slicer.

The first part of the AIS is the fore-optics which reimages the focal plane of the telescope on the slicing array. This is done with the proper magnification. In previous AIS, the magnification was made larger in the spectral direction than in the spatial (anamorphic magnification) so that a square spatial element on the sky (a spaxel) is imaged on the detector on one pixel in the spatial direction but 2 pixels in the spectral to get the proper sampling of the spectral element. This 
however comes with a reduction in the size of the field of view. It is not necessary with OCTOCAM because the seeing is typically many pixels wide so a slit of half the typical seeing is already more than 2 pixel wide.

The slicing mirror array is made of many long thin mirrors in the focal plane of the fore-optics that separate (slice) the field into the corresponding number of sub-images. Each mirror is tilted with a different angle than the others so that the beam is reflected in a different direction toward the pupil mirror array. Each slicing mirror is curved to image the pupil on or near the pupil mirror array. This solves a vignetting problem from previous designs of slicers. Each beam from a slicing mirror hit a different pupil mirror which images the slice on the slit. All the slices are then imaged side-by-side on the slit with a small gap between them to avoid cross-talks. The gap is 5 pixel wide in the present design. Superimposed to each slice images is a slit mirror that reimages the pupil at the right place in the spectrograph.

Two different designs were studied. In the first, the length of the slicer system is parallel to the telescope focal plane while it is parallel to the optical axis of the telescope in the second. The latter was chosen because it reduces the length of the slide supporting the focal plane instruments and because there was enough space along the optical axis in the OCTOCAM optical design.

\subsection{Length of slicer system along focal plane}

In this configuration, a fold on or near the focal plane rotates the beam from the telescope by $90^{\circ}$. The light then bounces between mirrors following paths not far from parallel to the focal plane (Figure 133). The present design is based on the GNIRS slicer design. It uses 2 off-axis aspheric mirrors as fore-optics to reimage the input field on the slicer. They should not be expensive considering the actual cost of aspheres and the small size of the mirrors. Also, it may not be necessary to use complex aspheres depending on the needed image quality in the focal and pupil planes. There is a pupil image between the 2 mirrors where a cold stop could be placed after slightly modifying the design to leave more space around the pupil image. At the slit, the slit mirrors reflect the light at $90^{\circ}$ into the spectrograph. They need to have a toroidal shape to give a sufficiently good pupil image quality. The slicing mirrors are all spherical with the same radius of curvature. The same is true for the pupil mirrors.

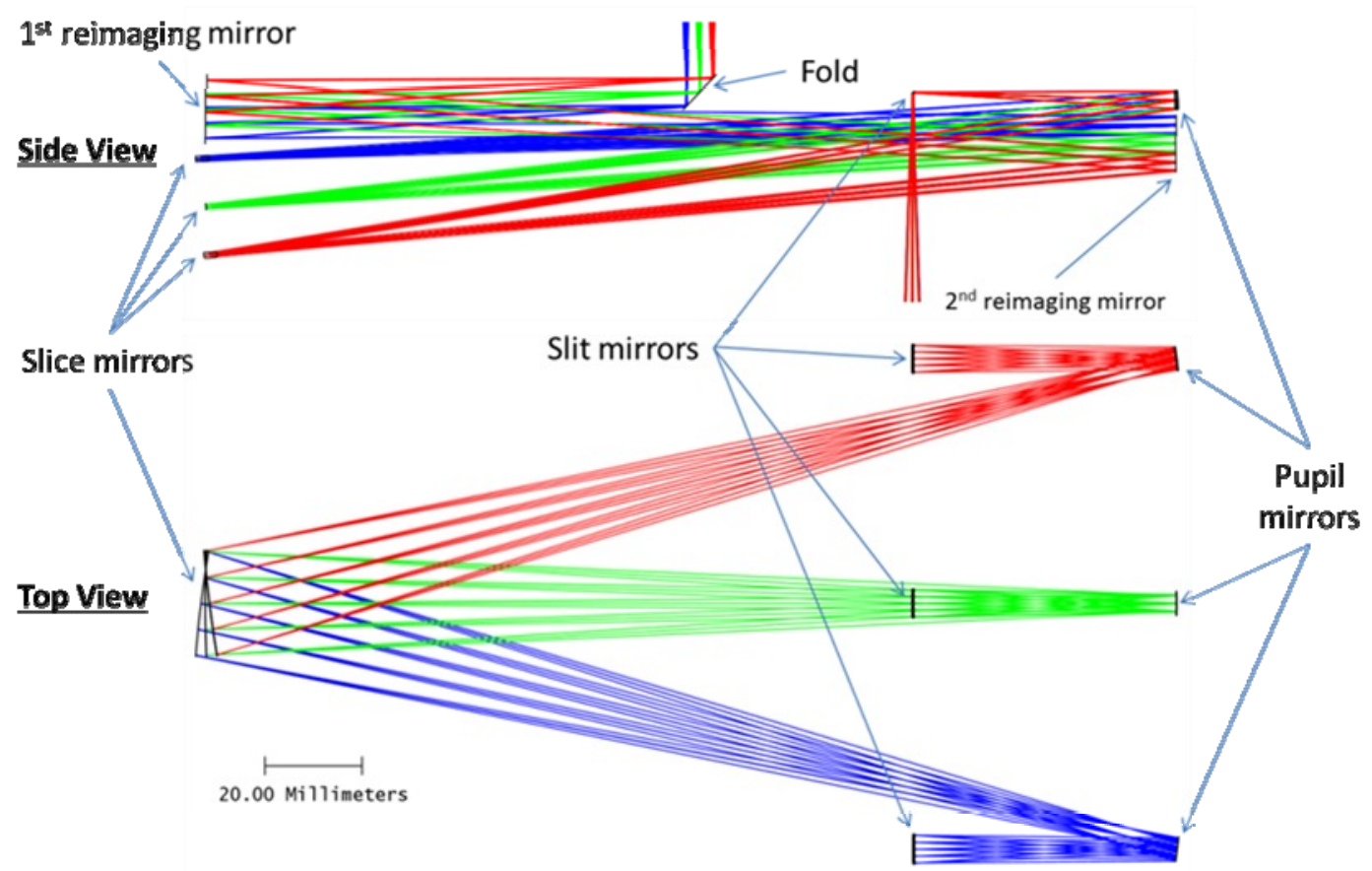

Figure 4. Layout of the slicer system with length parallel to the focal plane. Only central and edge slices are shown. 


\subsection{Length of slicer system along optical axis}

While there is less space along the optical axis than along the focal plane there is still enough room to put the slicer. In this case, there is no fold but there are three fore-optics mirrors instead of two (Figure 134). The second mirror is also used as a cold stop. The slit mirrors do not reflect the light at $90^{\circ}$ but with only a shallow angle. This makes it possible to make them spherical although a small asphere improves the pupil image quality.

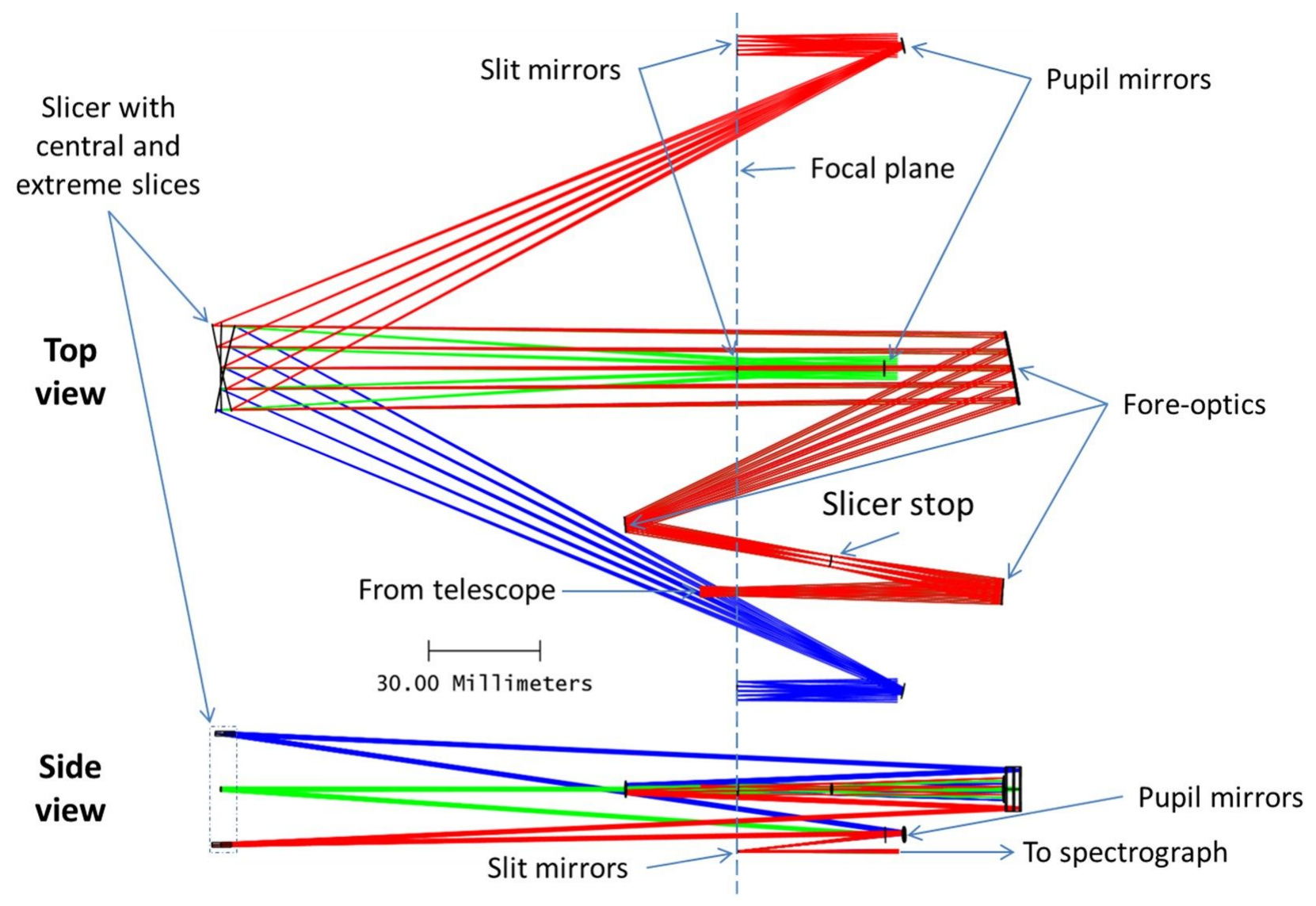

Figure 5. Layout of the slicer system with length parallel to the optical axis. Only central and edge slices are shown.

\subsection{Slicer for Adaptive Optics}

A slicer designed for AO would add that capability to OCTOCAM. It would be far more powerful than NIFS. The basic concept would be primarily for use with ALTAIR or GeMS so for the NIR spectrograph but a future more advanced AO system could extend the usable wavelength range to the VIS. The fore-optics would magnify anamorphically the field to make the spaxel width equal to the typical size of the ALTAIR PSF core, which is 0.08 ". This width would fit on 1 pixel in the spatial direction but the slices of 0.08 " would be magnified on 2 pixel in the spectral direction to get the proper minimum spectral sampling. Also, the magnification reduces the beam size entering the spectrograph from F/16 to F/36 $\mathrm{x} F / 72$. This reduction permits to use a slit length $60 \%$ longer with almost no modifications to the spectrograph for the following reasons:

- Only less than half of the detector's width is normally used in the spatial direction so the detectors can accommodate a longer slit. The detectors would then be better used with this slicer system. 
- The smaller beam permits to avoid vignetting except for the optics very much near the focal planes. Only the dichroic really needs an increase in diameter, which would be $15 \%$. The last 2 lenses of the NIR cameras (the smallest of the design) may need up to $2 \mathrm{~mm}$ increase in diameter.

- The smaller beam reduces considerably the aberrations. Combined with the ability to focus each slice separately when designing the slicer system the image quality is excellent with a typical PSF width (GEFWHM) of less than 0.5 pixel even if the spectrograph is not designed for such a long slit.

The AO slicer on OCTOCAM would have 31 slices of $0.08^{\prime \prime}$ x 3.6 " for a total field of $2.5^{\prime \prime}$ x $3.6 "$ ". It would have many advantages over NIFS:

- At least all 4 NIR bands $Y, J, H$ and $K_{S}$ would be observed simultaneously at a spectral resolution of about 5000 .

- The spaxel size of 0.08 " by 0.08 " would be a better fit to the ALTAIR PSF core of 0.08 " than the NIFS spaxel size of $0.10^{\prime \prime} \times 0.045^{\prime \prime}$ especially that a sampling of less than 1 pixel per FWHM can cause some undersampling problems even with dithering. With 2x2-images dithering the OCTOCAM AO slicer would give a full sampling of 2 spaxel per FWHM in both directions.

- The density of pixel per square arcsec is 1.4 times smaller which reduces the detector noise.

- While the field of view is the same than NIFS, the aspect ratio is better since a typical object would have a width and a length, not be circular or square. The longer length of 3.6" instead of the 3" of NIFS makes it more likely that an object would fit on the slicer.

As the cold stops in the cameras would be too large for the slower beam, the cold stop in the fore-optics would become the only cold stop. If optimized for GeMS, spaxels of 0.1", giving a larger field of 4.5" x 3.1", would be a better fit to the typical AO PSF. A field of view up to $30 \%$ larger in surface area could be obtained by using an even longer slit giving a field of up to 4.1 " x 2.8 " with 0.08 " spaxels. Additional oversizing of the dichroic, the last 2 small lenses of each NIR camera and the 2 lenses following the first dichroic would however be necessary.

The slice width would be approaching the size of the diffraction limit at the longest wavelength. This causes diffraction in the spectral direction which generates focal ratio degradation (FRD) of the beam. This problem was extensively studied for the GNIRS slicer. The pupil mirrors are consequently oversized. In the spectrograph, the beam is so slow (F/72) compared to the acceptance focal ratio of the spectrograph (F/16) that the additional FRD is negligible. NIFS and the JWST NIRSpec slicers have demonstrated that slicers of sufficient surface quality can be manufactured to work near the diffraction limit.

\subsection{IFU box}

To hold the slicers on the focal plane slide, a box attached to the slide will be manufactured. It will have to be longer than the standard boxes of the slide (Figure 6 left) to take advantage of the whole available space envelope. The box will use the sliding mechanism of the slide to select which of the 2 IFUs will be used (Figure 6 right) then avoiding an additional mechanism. The box has one entrance hole for each IFU on its front surface. Also, a baffle will block the parasite light on the back of the cryostat window. These 2 surfaces are near each other. By displacing the box $17 \mathrm{~mm}$ to the left from its centered position, one IFU hole is blocked by the window baffle while the other is in the beam. This selects one IFU. Moving the box $17 \mathrm{~mm}$ to the right instead will reverse which hole is blocked and which is in the beam then selecting the other IFU.

\subsection{Manufacturing}

Fabrication of the VIS+NIR slicer system would follow that for the ESI-IFU (at Keck) and MUSE (at the VLT). Both of these devices are all-spherical with a toroidal surface, operate in the visible/NIR, and are fabricated from Zerodur. Both systems used standard optical fabrication techniques using glass with a high degree of polish. MUSE optics were polished in groups, while ESI-IFU optics were polished individually. The MUSE slicer elements were wrung together (molecular adhesion), while ESI-IFU were glued with UV curing cement in an alignment jig. Both methods can in principle be cooled down to cryogenic temperatures. The ESI-IFU was successfully tested for use in NIR instrument by being plunged in liquid nitrogen while molecular adhesion should remain unchanged with temperature since the material is the same. Both options will be explored in the preliminary design stage. 
The AO slicer system is only for the NIR so the standard techniques used for other NIR slicers as in NIFS, KMOS and JWST NIRSpec can be used. Diamond turning was used in all slicer system optics of the first two while diamond rasterflight cutting was used in some optics of the NIRSpec slicer because their shapes did not have the symmetry necessary for diamond turning. This will not be the case with the AO slicer so diamond turning, which gives a better surface quality, will be used.
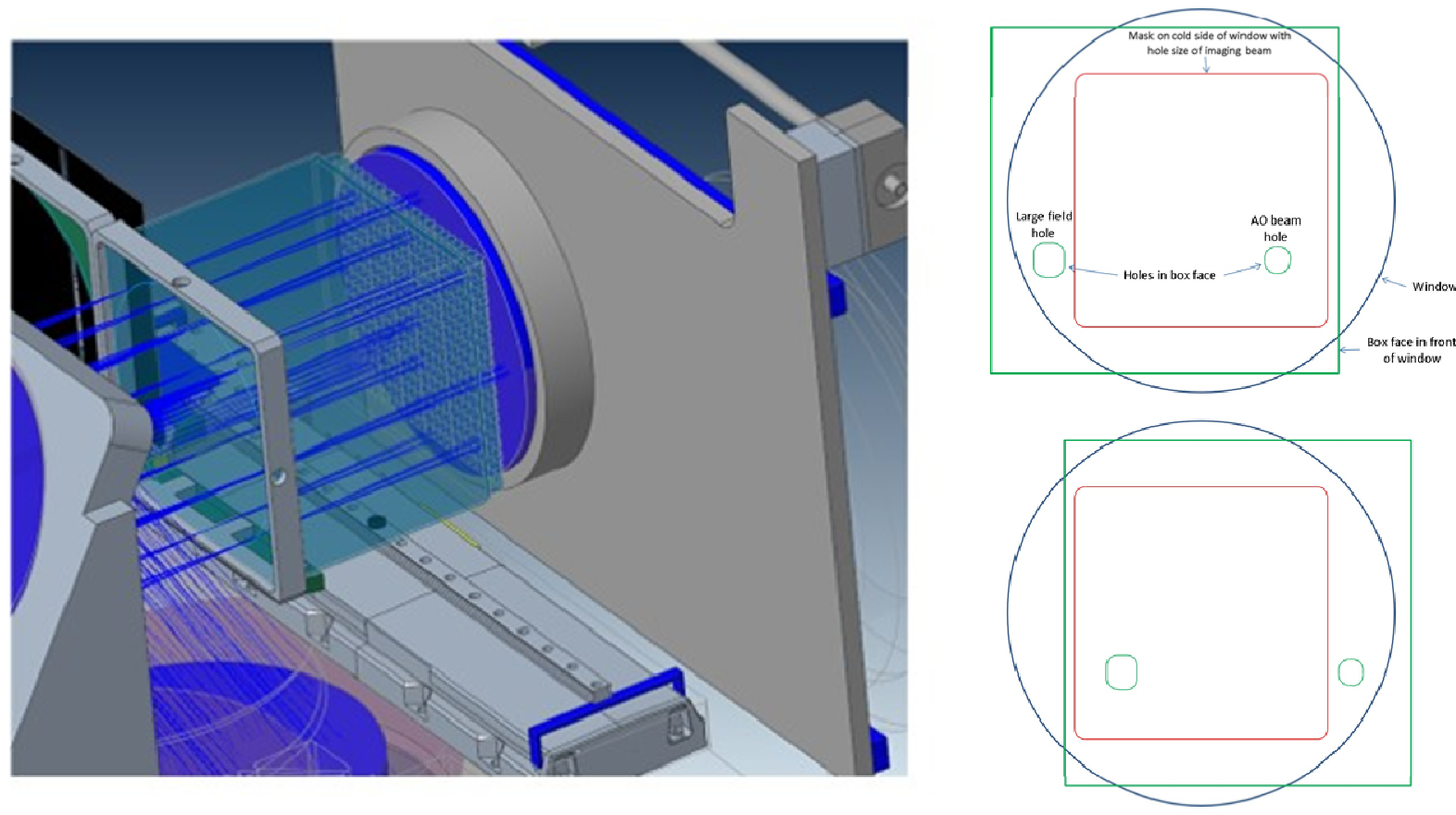

Figure 6. Standard box on focal plane slide behind cryostat window (left); slide movement left and right to select one IFU at a time (right).

\section{CONCLUSION}

OCTOCAM is the new imaging spectrograph being manufactured for Gemini. It will have 8 cameras covering the wavelength range from $370 \mathrm{~nm}$ to $2350 \mathrm{~nm}$ at a typical resolution of 3000-4000 depending of the wavelength. A slicer system will very significantly increase its capabilities. One slicer system will maximize the field size for observing in typical seeing over the whole wavelength range. It will have slices 0.4 " wide that gives spaxels of 0.18 " x 0.40 " and a field of nearly 10" x 7". This system will have far less readout noise than NIFS and the GMOS IFU. Another slicer system will take advantage of the Adaptive Optics system of Gemini with spaxels that have a size similar to the core size of the AO PSF. It will have spaxel $0.08 "$ x 0.08 " but a corresponding smaller field of $2.5 "$ x $3.6 "$. These IFUs will have a multitude of application, for example resolved studies of HII regions over a broad wavelength range and emission line galaxies over a broad redshift range using the same set of emission lines, observations of transients with only arcseconds localization very early without waiting for a sub-arcsecond position hence allowing to obtain very valuable early data. For bright transients such as SNe and GRBs we can study the immediate environment in detail, and even use the actual transient as AO tip-tilt star to study the environment at high spectral and very high angular resolutions.

\section{REFERENCES}

[1] F. Henault, R. Bacon, R. Content, B. Lantz, F. Laurent, J.-P. Lemonnier \& S. L. Morris, "Slicing the universe at affordable cost: the quest for the MUSE image slicer," Proc. SPIE 5249, 134-145 (2004).

[2] P. J. McGregor et al, "Gemini near-infrared integral field spectrograph (NIFS)," Proc. SPIE 4841, 1581-1591 (2003). 
[3] J. R. Allington-Smith, G. J. Murray, R. Content, G. N. Dodsworth, B. W. Miller, I. Jorgensen, I. Hook, "Integral field spectroscopy with the GEMINI multiobject spectrographs," Exp. Astron., 13, 1-37 (2002).

[4] J. Schmoll, G. N. Dodsworth, J. R. Allington-Smith, R. Content, G. J. Murray \& D. J. Robertson, "Design and construction of the IMACS-IFU, a 2000-element integral field unit," Proc. SPIE 5492, 624-633 (2004).

[5] J. R. Allington-Smith, C. M. Dubbeldam, R. Content, C. J. Dunlop, D. J. Robertson, J. Elias, B. Rodgers \& J. E. H. Turner, "An Image-Slicing Integral Field Unit for the Gemini Near-Infrared Spectrograph", Proc. SPIE 5492, 701-710 (2004).

[6] R. Content, "Optical design of the KMOS slicer system," Proc. SPIE 6269, id. 62693S (2006).

[7] J. J. Bryant et al, "SAMI: a new multi-object IFS for the Anglo-Australian Telescope," Proc. SPIE 8446, $84460 X(2012)$.

[8] J. Bryant et al, "Hector: a modular integral field spectrograph instrument for the Anglo-Australian Telescope," Proc. SPIE 10702, ***_*** (2018).

[9] R. Content, W. Saunders, J. Lawrence, J. Bryant \& R. Zhelem, "Optical design of the highly cost optimized Hector spectrograph," Proc. SPIE 10702, ***_*** (2018).

[10]R. Content, "Advanced Image Slicers for integral field spectroscopy with UKIRT and Gemini," Proc. SPIE 3354, 187-200 (1998).

[11]R. Content, "Advanced Image Slicers from the laboratory to NGST," Proc. ASP Conf. Series 195, 518-539 (2000).

[12] A. de Ugarte Postigo et al, "The OCTOCAM instrument concept at Gemini and beyond," 10702, ******* (2018).

[13] A. I. Sheinis, L. Laiterman, D. F. Hilyard, J. S. Miller, "Integral field unit for the echellette spectrograph and imager at Keck II," Proc. SPIE 4841, 1078-1085 (2003).

[14] C. M. Dubbeldam, D. J. Robertson, D. A. Ryder, R. M. Sharples, "Prototyping of diamond machined optics for the KMOS and JWST NIRSpec integral field units," Proc. SPIE, 6273, 62733F (2006).

[15] G. S. Wright et al, "Design and development of MIRI, the mid-IR instrument for JWST," SPIE, 7010, 70100T (2008). 